\title{
Kapitza thermal resistance across individual grain boundaries in graphene
}

\author{
Khatereh Azizi $^{\mathrm{a}, \mathrm{b}}$, Petri Hirvonen ${ }^{\mathrm{b}}$, Zheyong Fan $^{\mathrm{b}, *}$, Ari Harju ${ }^{\mathrm{b}}$, Ken R. Elder ${ }^{\mathrm{c}}$, Tapio Ala-Nissila ${ }^{\mathrm{b}, \mathrm{d}}$, S. Mehdi Vaez Allaei ${ }^{\mathrm{a}, \mathrm{e}}$ \\ ${ }^{a}$ Department of Physics, University of Tehran, Tehran 14395-547, Iran \\ ${ }^{b}$ COMP Centre of Excellence, Department of Applied Physics, Aalto University School of Science, P.O. Box 11000, FIN-00076 Aalto, Espoo, Finland \\ ${ }^{c}$ Department of Physics, Oakland University, Rochester, Michigan 48309, USA \\ ${ }^{d}$ Departments of Mathematical Sciences and Physics, Loughborough University, Loughborough, Leicestershire LE11 3TU, UK \\ ${ }^{e}$ School of Physics, Institute for Research in Fundamental Sciences (IPM), Tehran 19395-5531, Iran
}

\begin{abstract}
We study heat transport across individual grain boundaries in suspended monolayer graphene using extensive classical molecular dynamics (MD) simulations. We construct bicrystalline graphene samples containing grain boundaries with symmetric tilt angles using the two-dimensional phase field crystal method and then relax the samples with MD. The corresponding Kapitza resistances are then computed using nonequilibrium MD simulations. We find that the Kapitza resistance depends strongly on the tilt angle and shows a clear correlation with the average density of defects in a given grain boundary, but is not strongly correlated with the grain boundary line tension. We also show that quantum effects are significant in quantitative determination of the Kapitza resistance by applying the mode-by-mode quantum correction to the classical MD data. The corrected data are in good agreement with quantum mechanical Landauer-Bütticker calculations.
\end{abstract}

Keywords: Grain boundary, Kapitza resistance, Graphene, Molecular dynamics, Phase field crystal

\section{Introduction}

Graphene [1], the famous two-dimensional allotrope of carbon, has been demonstrated to have extraordinary electronic [2], mechanical [3], and thermal [4] properties in its pristine form. However, large-scale graphene films, which are needed for industrial applications are typically grown by chemical vapor deposition [5] and are polycrystalline in nature [6], consisting of domains of pristine graphene with varying orientations separated by grain boundaries (GB) [7-9]. They play a significant or even dominant role in influencing many properties of graphene [10, 11].

One of the most striking properties of pristine graphene is its extremely high heat conductivity, which has been shown to be in excess of $5000 \mathrm{~W} / \mathrm{mK}$ [4, 12]. Grain boundaries in graphene act as line defects or one-dimensional interfaces which leads to a strong reduction of the heat conductivity in multigrain samples [13, 14]. The influence of GBs can be quantified by the Kapitza or thermal boundary resistance $R$. The Kapitza resistance of graphene grain boundaries has been previously computed using molecular dynamics (MD) [15, 16] and LandauerBütticker [17, 18] methods, and has also been measured experimentally [19]. However, these works have only considered a few separate tilt angles, and a systematic investigation on the dependence of the Kapitza resistance on the tilt angle between any two pristine grains is still lacking. The relevant questions here concern both the magnitude $R$ for different tilt angles and

\footnotetext{
${ }^{*}$ Corresponding author. E-mail: brucenju@gmail.com (Zheyong Fan) Email addresses: petri.hirvonen@aalto.fi (Petri Hirvonen), smvaez@ut.ac.ir (S. Mehdi Vaez Allaei)
}

possible correlations between the structure or line tension of the GBs and the corresponding value of $R$.

Modelling realistic graphene GBs has remained a challenge due to the multiple length and time scales involved. Recently, an efficient multiscale approach [20] for modelling polycrystalline graphene samples was developed based on phase field crystal (PFC) models [21, 22]. The PFC models are a family of continuum methods for modelling the atomic level structure and energetics of crystals, and their evolution at diffusive time scales (as compared to vibrational time scales in MD). The PFC models retain full information about the atomic structure and elasticity of the solid [22]. It has been shown [20] that using the PFC approach in two-dimensional space one can obtain large, realistic and locally relaxed microstructures that can be mapped to atomic coordinates for further relaxation in threedimensional space with the usual atomistic simulation methods.

In this work, we employ the multiscale PFC strategy of Ref. [20] to generate large samples of tilted, bicrystalline graphene with a well-defined GB between the two grains. These samples are then further relaxed with MD at $T=300 \mathrm{~K}$. A heat current is generated across the bicrystals using nonequilibrium MD (NEMD) simulations, and the Kapitza resistance is computed from the temperature drop across the GB. We map the values of $R(\theta)$ for a range of different tilt angles $\theta$ and demonstrate how $R$ correlates with the structure of the GBs. Finally, we demonstrate that quantum corrections need to be included in $R$ to obtain quantitative agreement with experiments and lattice dynamical calculations. 


\section{Models and Methods}

\subsection{PFC models}

PFC approaches typically employ a classical density field $\psi(\boldsymbol{r})$ to describe the systems. The ground state of $\psi$ is governed by a free energy functional $F[\psi(\boldsymbol{r})]$ that is minimized either by a periodic or a constant $\psi$, corresponding to crystalline and liquid states, respectively. We use the standard PFC model

$$
F=\int d \boldsymbol{r}\left(\frac{1}{2} \psi\left[\epsilon+\left(q^{2}+\nabla^{2}\right)^{2}\right] \psi+\frac{1}{3} \tau \psi^{3}+\frac{1}{4} \psi^{4}\right)
$$

where the model parameters $\epsilon$ and $\tau$ are phenomenological parameters related to temperature and average density, respectively. The component $\left(q^{2}+\nabla^{2}\right)^{2}$ penalizes for deviations from the length scale set by the wave number $q$, giving rise to a spatially oscillating $\psi$ and to elastic behaviour [21,22]. The crystal structure in the ground state is dictated by the formulation of $F$ and the average density of $\psi$, and for certain parameter values the ground state of $\psi$ displays a honeycomb lattice of density maxima as appropriate for graphene [20].

The PFC calculations are initialized with symmetrically tilted 2-crystals in a periodic, two-dimensional computational unit cell. The initial guess for the crystalline grains is obtained by using the one-mode approximation [22]

$$
\psi(x, y)=\cos (q x) \cos \left(\frac{q y}{\sqrt{3}}\right)-\frac{1}{2} \cos \left(\frac{2 q y}{\sqrt{3}}\right),
$$

and by rotating alternatingly by $\pm \theta$. The tilt angle between two adjacent grains is $\theta-(-\theta)=2 \theta$, which ranges from $2 \theta=0^{\circ}$ to $2 \theta=60^{\circ}$ (see Fig. 1 for examples). We consider a subset of the tilt angles investigated in Ref. [20], with the exact values being listed in Table 1 . The rotated grains and the unit cell size are matched together as follows: if just one of the rotated grains filled the whole unit cell, it would be perfectly continuous at the periodic edges. Along both interfaces, narrow strips a few atomic spacings wide are set to the average density - corresponding to a disordered state - to give the grain boundaries some additional freedom to find their lowest-energy configuration. We assume non-conserved dynamics to relax the systems in analogy to chemical vapour deposition [23] - the number of atoms in the monolayer can vary as if due to exchange with a vapor phase. In addition, the unit cell dimensions are allowed to vary to minimize strain. Further details of the PFC calculations can be found in Ref. [20]. The relaxed density field is mapped to a discrete set of atomic coordinates suited for the initialization of MD simulations [20].

\subsection{NEMD simulations}

We use the NEMD method as implemented in the GPUMD (graphics processing units molecular dynamics) code [24-26] to calculate the Kapitza resistance, using the Tersoff [27] potential with optimized parameters [28] for graphene. The initial structures obtained by the PFC method are rescaled by an appropriate factor to have zero in-plane stress at $300 \mathrm{~K}$ in the MD simulations with the optimized Tersoff potential [28].
In the NEMD simulations, periodic boundary conditions are applied in the transverse direction, whereas fixed boundary conditions are applied in the transport direction. We first equilibrate the system at $1 \mathrm{~K}$ for $1 \mathrm{~ns}$, then increase the temperature from 1 $\mathrm{K}$ to $300 \mathrm{~K}$ during $1 \mathrm{~ns}$, and then equilibrate the system at 300 $\mathrm{K}$ for $1 \mathrm{~ns}$. After these steps, we apply a Nosé-Hoover chain of thermostats [29-31] to the heat source and sink, choosing as two blocks of atoms around the two ends of the system, as schematically shown in Fig. 2. The temperatures of the heat source and sink are maintained at $310 \mathrm{~K}$ and $290 \mathrm{~K}$, respectively. We have checked that steady state can be well established within $5 \mathrm{~ns}$. In view of this, we calculate the temperature profile $T(x)$ of the system and the energy exchange rate $Q$ between the system and the thermostats using data sampled in another $5 \mathrm{~ns}$. The velocity-Verlet integration scheme [32] with a time step of $1 \mathrm{fs}$ is used for all the calculations. Three independent calculations are performed for each system and the error estimates reported in Table 1 correspond to the standard error of the independent results.

In steady state, apart from the nonlinear regions around the heat source and the sink intrinsic to the method, a linear temperature profile can be established on each side of the GB, but with an inherent discontinuity (temperature jump) at the GB. An example of this for the system with $2 \theta=9.43^{\circ}$ is shown in Fig. 3. The Kapitza resistance $R$ is defined as the ratio of the temperature jump $\Delta T$ and the heat flux $J$ across the grain boundary:

$$
R=\frac{\Delta T}{J}
$$

where $J$ can be calculated from the energy exchange rate $Q$ (between the system and thermostat) and the cross-sectional area $S$ (graphene thickness is chosen as $0.335 \mathrm{~nm}$ in our calculations), i.e. $J=Q / S$.

\section{Results and Discussion}

It is well known [15, 16, 33] that the calculated Kapitza resistance depends on the sample length in NEMD simulations. Figure 4 shows the calculated Kapitza resistance $R$ in the $2 \theta=9.43^{\circ}$ case as a function of the sample length $L_{x}$. Using fixed boundary conditions as described above, $R$ saturates at around $L_{x}=400 \mathrm{~nm}$. On the other hand, using periodic boundaries as described in Ref. [15], $R$ converges more slowly. To this end, we have here used fixed boundary conditions and a sample length of $400 \mathrm{~nm}$ for all the systems. The calculated temperature jump $\Delta T$, heat flux $J$, and Kapitza resistance $R$ in the 13 bicrystalline systems are listed in Table 1

The Kapitza resistance calculated from the heat flux does not contain any information on the contributions from individual phonon modes. Methods of spectral decomposition of both the heat current (flux) [37-42] and the temperature [43] within the NEMD framework have been developed recently. Here, we use the spectral decomposition formalism as described in Ref. [42] to calculate the spectral conductance $g(\omega)$ of the $2 \theta=9.43^{\circ}$ system. In this method, one first calculates the following nonequilibrium heat current correlation function $(t$ is the correlation 


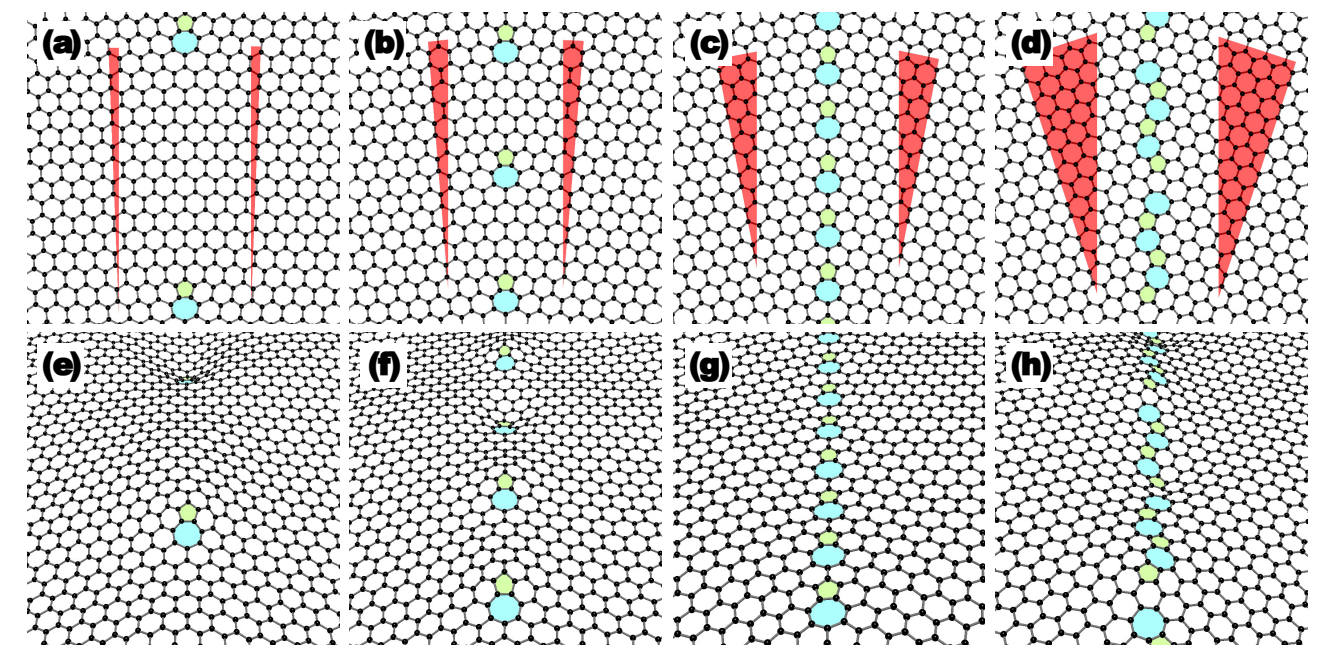

Figure 1: A schematic illustration of the definition of the tilt angle in bicrystalline graphene with symmetrically tilted grain boundaries. (a)(d): Grain boundary with tilt angles (indicated by the red wedges) of $2 \theta=4.42^{\circ}, 9.43^{\circ}, 21.79^{\circ}$, and $36.52^{\circ}$ before MD relaxation. (e)-(h): the corresponding systems after MD relaxation. For clarity, only the small part close to the grain boundary is shown for each system; the sample size in our NEMD simulations is much larger, which is $L_{x}=400 \mathrm{~nm}$ (transport direction) and $L_{y}=25 \mathrm{~nm}$ (transverse direction). (A colour version of this figure can be viewed online.)

Table 1: The GB tilt angle $2 \theta$, the corresponding temperature jump $\Delta T$, heat flux $J$, Kapitza resistance $R$, Kapitza length $L_{K}$, grain boundary line tension $\gamma$, and defect density $\rho$ for the 13 bicrystalline graphene samples considered here.

\begin{tabular}{rrrrrrr}
\hline \hline $2 \theta\left(^{\circ}\right)$ & $\Delta T(\mathrm{~K})$ & $J\left(\mathrm{GW} / \mathrm{m}^{2}\right)$ & $R\left(\mathrm{~m}^{2} \mathrm{~K} / \mathrm{GW}\right)$ & $L_{K}(\mathrm{~nm})$ & $\gamma(\mathrm{eV} / \mathrm{nm})$ & $\rho(1 / \mathrm{nm})$ \\
\hline 1.10 & $0.09 \pm 0.07$ & $51.3 \pm 0.4$ & $0.0018 \pm 0.0013$ & $10 \pm 7$ & 0.55 & 0.08 \\
4.41 & $0.73 \pm 0.14$ & $50.4 \pm 0.6$ & $0.0144 \pm 0.0026$ & $75 \pm 13$ & 2.21 & 0.31 \\
9.43 & $1.36 \pm 0.15$ & $45.7 \pm 0.3$ & $0.0298 \pm 0.0033$ & $155 \pm 17$ & 3.84 & 0.67 \\
13.17 & $1.62 \pm 0.08$ & $48.1 \pm 0.5$ & $0.0337 \pm 0.0020$ & $175 \pm 10$ & 4.71 & 0.93 \\
18.73 & $1.99 \pm 0.06$ & $43.9 \pm 0.2$ & $0.0453 \pm 0.0015$ & $236 \pm 8$ & 5.02 & 1.32 \\
21.79 & $1.97 \pm 0.01$ & $47.8 \pm 0.2$ & $0.0412 \pm 0.0004$ & $214 \pm 2$ & 4.69 & 1.54 \\
27.80 & $2.39 \pm 0.04$ & $43.6 \pm 0.3$ & $0.0548 \pm 0.0005$ & $285 \pm 3$ & 4.71 & 1.95 \\
32.20 & $2.49 \pm 0.10$ & $43.4 \pm 0.7$ & $0.0574 \pm 0.0033$ & $298 \pm 17$ & 3.77 & 2.25 \\
36.52 & $2.48 \pm 0.14$ & $43.9 \pm 0.5$ & $0.0565 \pm 0.0026$ & $294 \pm 14$ & 4.93 & 1.91 \\
42.10 & $2.30 \pm 0.02$ & $43.1 \pm 0.2$ & $0.0534 \pm 0.0006$ & $278 \pm 3$ & 5.50 & 1.46 \\
46.83 & $1.93 \pm 0.06$ & $46.4 \pm 0.3$ & $0.0416 \pm 0.0014$ & $216 \pm 7$ & 5.16 & 1.06 \\
53.60 & $1.01 \pm 0.06$ & $41.2 \pm 0.3$ & $0.0245 \pm 0.0014$ & $127 \pm 7$ & 3.36 & 0.52 \\
59.04 & $0.17 \pm 0.07$ & $47.3 \pm 0.1$ & $0.0036 \pm 0.0014$ & $19 \pm 7$ & 0.61 & 0.08 \\
\hline \hline
\end{tabular}

time):

$$
K(t)=\sum_{i \in A} \sum_{j \in B}\left\langle\frac{\partial U_{i}(0)}{\partial \vec{r}_{i j}} \cdot \vec{v}_{j}(t)-\frac{\partial U_{j}(0)}{\partial \vec{r}_{j i}} \cdot \vec{v}_{i}(t)\right\rangle,
$$

where $U_{i}$ and $\vec{v}_{i}$ are respectively the potential energy and velocity of particle $i, \vec{r}_{i j}=\vec{r}_{j}-\vec{r}_{i}\left(\vec{r}_{i}\right.$ is the position of particle $\left.i\right)$, and $K(t=0)$ measures the heat current flowing form a block $A$ to an adjacent block $B$ arranged along the transport direction. Then, one performs a Fourier transform to get the spectral conductance:

$$
g(\omega)=\frac{2}{S \Delta T} \int_{-\infty}^{+\infty} d t e^{i \omega t} K(t) .
$$

The spectral conductance is normalized as

$$
G=\int_{0}^{\infty} \frac{d \omega}{2 \pi} g(\omega)
$$

where $G$ is the total Kapitza conductance (also called thermal boundary conductance), which is the inverse of the Kapitza resistance $G=1 / R$.

Figure 5 (a) shows the calculated correlation function $K(t)$, which resembles the velocity autocorrelation function whose Fourier transform is the phonon density of states [44]. Indeed, thermal conductance in the quasi-ballistic regime is intimately related to the phonon density of states. The corresponding spectral conductance $g(\omega)$ is shown as the solid line in Fig. 5(b). The total thermal boundary conductance is $G \approx 33 \mathrm{GW} / \mathrm{m}^{2} / \mathrm{K}$, corresponding to a Kapitza resistance of $R \approx 0.03 \mathrm{~m}^{2} \mathrm{~K} / \mathrm{GW}$.

In view of the high Debye temperature (around $2000 \mathrm{~K}$ ) for pristine graphene [45], we expect that it is necessary to correct the classical results to properly account for possible quantum effects. While using classical statistics can lead to [46] an underestimate of the scattering time for the low-frequency 


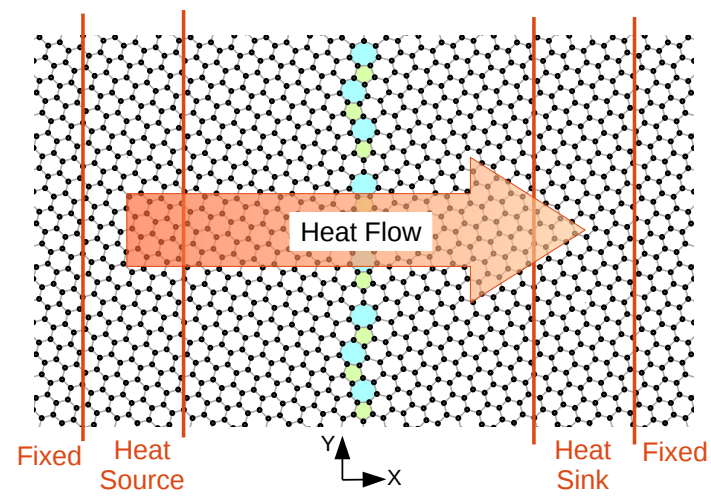

Figure 2: A schematic illustration of the NEMD setup used in computing the Kapitza resistance. (A colour version of this figure can be viewed online.)

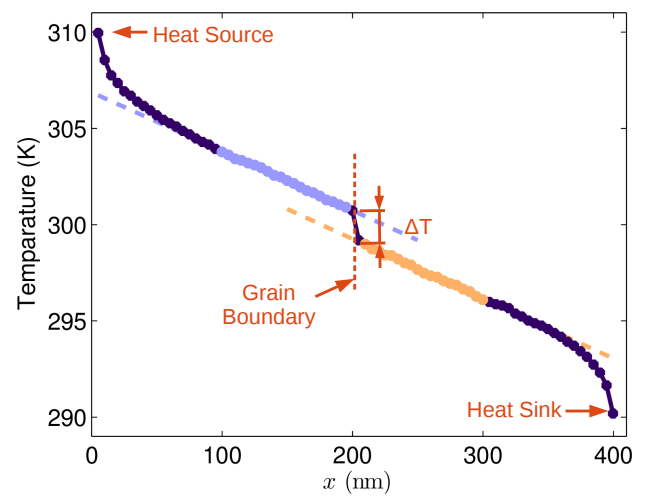

Figure 3: A typical steady-state temperature profile in bicrystalline graphene with a tilt angle of $2 \theta=9.43^{\circ}$. On each side of the grain boundary, excluding the nonlinear region around the heat source or the sink, one can fit the temperature by a linear function and then extract the temperature jump as the difference between the two linear functions at the grain boundary [15, 16, 33, 36]. (A colour version of this figure can be viewed online.)

phonons as well as an overestimate of the heat capacity of the high-frequency phonons for thermal transport in the diffusive regime, only the second effect matters here in the quasi-ballistic regime. Therefore, one can correct the results by multiplying the classical spectral conductance by the ratio of the quantum heat capacity to the classical one: $x^{2} e^{x} /\left(e^{x}-1\right)^{2}$, where $x=\hbar \omega / k_{B} T$, with $\hbar, k_{B}, T$ being the Planck constant, Boltzmann constant, and system temperature, respectively. This factor is unity in the low-frequency (high-temperature) limit and zero in the high-frequency (low-temperature) limit. Applying this mode-to-mode quantum correction to the classical spectral conductance gives the quantum spectral conductance represented by the dashed line in Fig. 5(b). The integral of the quantum corrected spectral conductance is reduced by a factor of about 2.3 as compared to the classical one.

Figure 6(a) shows the calculated Kapitza resistances for all the 13 systems as a function of the tilt angle, both before (red squares) and after (blue circles) applying the quantum correction. It clearly shows that the Kapitza resistance depends

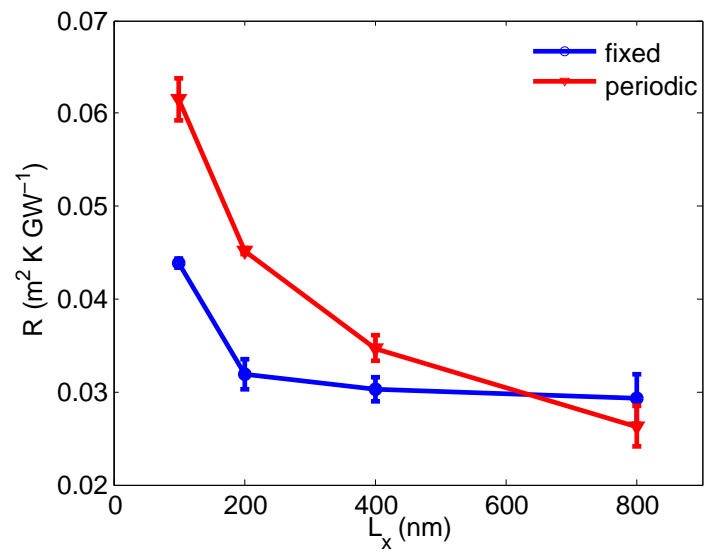

Figure 4: Kapitza resistance as a function of the sample length $L_{x}$ obtained by using fixed boundary conditions (circles) and periodic boundaries (triangles) in the case of $2 \theta=9.43^{\circ}$. (A colour version of this figure can be viewed online.)

strongly on the tilt angle, varying by more than one order of magnitude. The Kapitza resistance increases monotonically from both sides to the middle angle of $2 \theta \sim 30^{\circ}$, except for one "anomalous" system with $2 \theta=21.79^{\circ}$. This system has smaller $R$ than that with $2 \theta=18.73^{\circ}$. One intuitive explanation is that this system is relatively flat compared to other systems, as can been seen from Figs. 11.e)-(h). Similar "anomalous" heat transport has been reported in Ref. [47] for the same grain boundary tilt angle.

The largest Kapitza resistances occurring around the intermediate angles, being about $0.12 \mathrm{~m}^{2} / \mathrm{K} / \mathrm{GW}$ after quantum corrections, are more than an order of magnitude smaller than those in grain boundaries in silicon nanowires [36]. A more reasonable comparison between different materials is in terms of the Kapitza length $L_{K}$ [48], defined as the system length of the corresponding pristine material at which the bulk thermal resistance due to phonon-phonon scattering equals the Kapitza resistance. Mathematically, we have

$$
L_{K}=\kappa R,
$$

where $\kappa$ is the thermal conductivity of the bulk material. We calculate $L_{K}$ by assuming a value of $\kappa=5200 \mathrm{~W} / \mathrm{mK}$ for pristine graphene according to the very recent experiments [12] and list the values in Table 1 The largest Kapitza lengths (corresponding to the largest Kapitza resistances) before quantum corrections are about $300 \mathrm{~nm}$, which would be about $700 \mathrm{~nm}$ after quantum corrections. These values are actually larger than those for silicon nanowires. Therefore, the effect of grain boundaries on heat transport in graphene is not small even though the Kapitza resistances are relatively small.

To facilitate comparison with previous works, we also show the Kapitza conductances in Fig. 6(b). The Kapitza conductances in our systems range from about $17 \mathrm{GW} / \mathrm{m}^{2} / \mathrm{K}$ to more than $500 \mathrm{GW} / \mathrm{m}^{2} / \mathrm{K}$ before applying the quantum corrections. Bagri et al. [15] reported Kapitza conductance values (obtained by NEMD simulations with periodic boundary conditions in the transport direction) ranging from $15 \mathrm{GW} / \mathrm{m}^{2} / \mathrm{K}$ to 
(a)
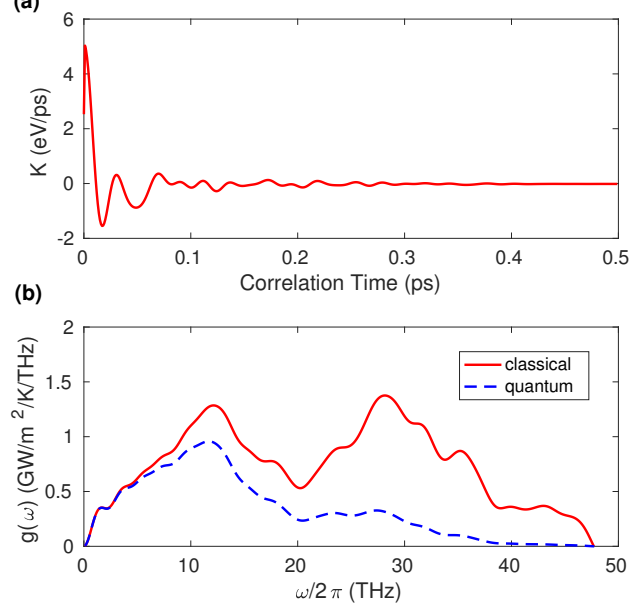

Figure 5: (a) The nonequilibrium heat current correlation function $K(t)$ as a function of correlation time. (b) The spectral conductance before (solid line) and after (dashed line) mode-to-mode quantum corrections as a function of the phonon frequency. The system considered here corresponds to the case of $2 \theta=9.43^{\circ}$, but similar results are obtained for all other cases. (A colour version of this figure can be viewed online.)

$45 \mathrm{GW} / \mathrm{m}^{2} / \mathrm{K}$. The lower limit of $15 \mathrm{GW} / \mathrm{m}^{2} / \mathrm{K}$ does not conflict with our data, as this value is reported in a system of a grain size of $25 \mathrm{~nm}$, where the data cannot have converged yet. On the other hand, Cao and Qu (obtained by NEMD simulations with fixed boundary conditions in the transport direction) [16] reported saturated Kapitza conductance values in the range of $19-47 \mathrm{GW} / \mathrm{m}^{2} / \mathrm{K}$, which fall well within the values that we obtained. Last, we note that quantum mechanical LandauerBütticker calculations by Serov et al. [18] predicted the Kapitza conductance to be about $8 \mathrm{GW} / \mathrm{m}^{2} / \mathrm{K}$ for graphene grain boundaries comparable to those in our samples with intermediate tilt angles $\left(2 \theta \sim 30^{\circ}\right)$. This is much smaller than the classical Kapitza conductances (about $20 \mathrm{GW} / \mathrm{m}^{2} / \mathrm{K}$ ), but agree well with our quantum corrected values. This comparison justifies the mode-to-mode quantum correction we applied to the classical data and resolves the discrepancy between the results from classical NEMD simulations and quantum mechanical LandauerBütticker calculations.

The last remaining issue concerns the possible correlation of the values of $R(\theta)$ with the energetics and structure of the GBs. The grain boundary line tension and the defect density are closely related to the tilt angle. The line tension $\gamma$ is defined as

$$
\gamma=\lim _{L_{y} \rightarrow \infty} \frac{\Delta E}{L_{y}},
$$

in the thermodynamic limit, where $\Delta E$ is the formation energy for a GB of length $L_{y}$. The defect density is defined as

$$
\rho=\frac{N_{\mathrm{p}-\mathrm{h}}}{L_{y}},
$$

where $N_{\mathrm{p}-\mathrm{h}}$ is the number of pentagon-heptagon pairs in the grain boundary. The calculated $\gamma$ and $\rho$ values for all the tilt an-

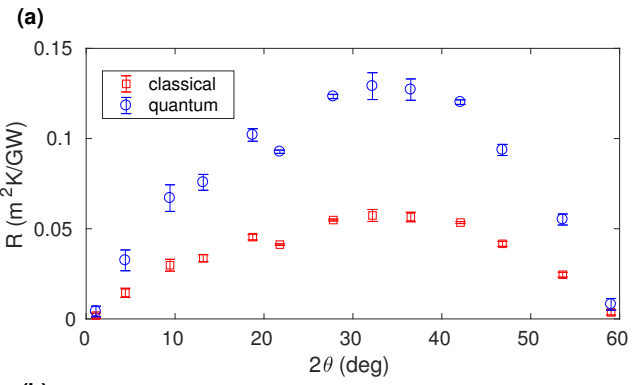

(b)

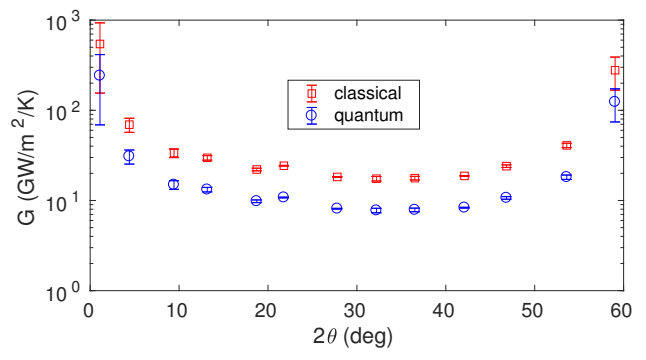

Figure 6: (a) Kapitza resistance $R$ of the grain boundary before (labeled as "classical") and after (labeled as "quantum") the mode-tomode quantum correction as a function of the tilt angle $2 \theta$. (b) The corresponding Kapiza conductance $G$ as a function of the tilt angle. (A colour version of this figure can be viewed online.)

gles are listed in Table 1 and plotted in Figs. 7(a)-(b). In Figs. 7(c)-(d), we plot the Kapitza resistance against $\gamma$ and $\rho$, respectively. At small and large tilt angles, where the defect density is relatively small, there is a clear linear dependence of $R$ on both $\gamma$ and $\rho$. However, at intermediate tilt angles $\left(2 \theta \approx 30^{\circ}\right)$, where the defect density is relatively large, the linear dependences become less clear, especially between $R$ and $\gamma$, which may indicate increased interactions between the defects. Overall, there is a stronger correlation between the Kapitza resistance and the defect density which is consistent with the idea of enhanced phonon scattering with increasing $\rho$.

\section{Summary and Conclusions}

In summary, we have employed an efficient multiscale modeling strategy based on the PFC approach and atomistic MD simulations to systematically evaluate the Kapitza resistances in graphene grain boundaries for a wide range of tilt angles between adjacent grains. Strong correlations between the Kapitza resistance and the tilt angle, the grain boundary line tension, and the defect density are identified. Quantum effects, which have been ignored in previous studies, are found to be significant. By applying a mode-to-mode quantum correction method based on spectral decomposition, we have demonstrated that good agreement between the classical molecular dynamics data and the quantum mechanical Landauer-Bütticker method can be obtained.

We emphasize that we have only considered suspended systems in this work. In a recent experimental work by Yasaei $e t$ al. [19], Kapitza conductances (inverse of the Kapitza resis- 

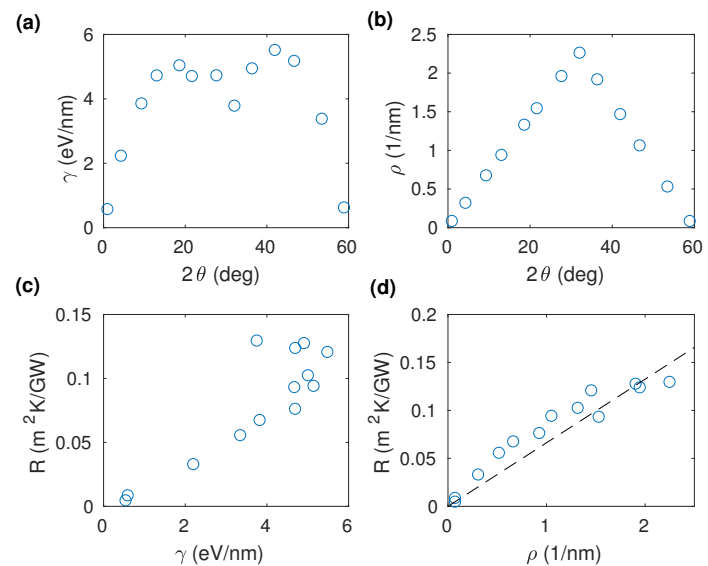

Figure 7: (a) The grain boundary line tension $\gamma$ and (b) the defect density $\rho$ versus the tilt angle $2 \theta$. (c) and (d) The quantum corrected Kapitza resistance $R$ versus $\gamma$ and $\rho$, respectively. The dashed line in (d) is a guide to the eye. (A colour version of this figure can be viewed online.)

tance) for a few supported (on $\mathrm{SiN}$ substrate) samples containing grain boundaries with different tilt angles were measured. The Kapitza conductances reported in this work are about one order of magnitude smaller than our quantum corrected values. This large discrepancy indicates that certain substrates may strongly affect heat transport across graphene grain boundaries and more work is needed to clarify this.

\section{Acknowledgements}

This research has been supported by the Academy of Finland through its Centres of Excellence Program (Project No. 251748). We acknowledge the computational resources provided by Aalto Science-IT project and Finland's IT Center for Science (CSC). K.A. acknowledges financial support from the Ministry of Science and Technology of Islamic Republic of Iran. P.H. acknowledges financial support from the Foundation for Aalto University Science and Technology, and from the Vilho, Yrjö and Kalle Väisälä Foundation of the Finnish Academy of Science and Letters. Z.F. acknowledges the support of the National Natural Science Foundation of China (Grant No. 11404033). K.R.E. acknowledges financial support from the National Science Foundation under Grant No. DMR1506634 .

\section{References}

[1] K. S. Novoselov, A. K. Geim, S. V. Morozov, D. Jiang, Y. Zhang, S. V. Dubonos, I. V. Grigorieva, A. A. Firsov, Electric field effect in atomically thin carbon films Science 306 (5696) (2004) 666669. arXiv:http://science.sciencemag.org/content/306/ 5696/666.full.pdf doi:10.1126/science.1102896 URL http://science.sciencemag.org/content/306/5696/666

[2] A. H. Castro Neto, F. Guinea, N. M. R. Peres, K. S. Novoselov, A. K. Geim, The electronic properties of graphene Rev. Mod. Phys. 81 (2009) 109-162. doi:10.1103/RevModPhys.81.109 URL https://link.aps.org/doi/10.1103/RevModPhys.81.109
[3] C. Lee, X. Wei, J. W. Kysar, J. Hone, Measurement of the elastic properties and intrinsic strength of monolayer graphene Science 321 (5887) (2008) 385-388. arXiv:http://science.sciencemag. org/content/321/5887/385.full.pdf doi:10.1126/science. 1157996

URL http://science.sciencemag.org/content/321/5887/385

[4] A. A. Balandin, S. Ghosh, W. Bao, I. Calizo, D. Teweldebrhan, F. Miao, C. N. Lau, Superior thermal conductivity of single-layer graphene Nano Letters 8 (3) (2008) 902-907. arXiv: http://dx.doi.org/10.1021/ nl0731872 doi:10.1021/nl0731872

URL http://dx.doi.org/10.1021/nl0731872

[5] X. Li, W. Cai, J. An, S. Kim, J. Nah, D. Yang, R. Piner, A. Velamakanni, I. Jung, E. Tutuc, S. K. Banerjee, L. Colombo, R. S. Ruoff, Large-area synthesis of high-quality and uniform graphene films on copper foils Science 324 (5932) (2009) 1312-1314. arXiv:http:// science.sciencemag.org/content/324/5932/1312.full.pdf doi:10.1126/science.1171245

URL http://science.sciencemag.org/content/324/5932/ 1312

[6] P. Huang, C. Ruiz-Vargas, A. V. D. Zande, W. Whitney, M. Levendorf, J. Kevek, S. Garg, J. Alden, C. Hustedt, Y. Zhu, J. Park, P. McEuren, D. Muller, Grains and grain boundaries in single-layer graphene atomic patchwork quilts Nature 469 (2011) $389-392$.

URL http://dx.doi.org/10.1038/nature09718

[7] O. V. Yazyev, S. G. Louie, Topological defects in graphene: Dislocations and grain boundaries Phys. Rev. B 81 (2010) 195420. doi:10.1103/PhysRevB.81.195420

URL https://link.aps.org/doi/10.1103/PhysRevB.81. 195420

[8] T.-H. Liu, G. Gajewski, C.-W. Pao, C.-C. Chang, Structure, energy, and structural transformations of graphene grain boundaries from atomistic simulations Carbon 49 (7) (2011) $2306-2317$. doi:http://dx.doi.org/10.1016/j.carbon.2011.01.063 URL http://www.sciencedirect.com/science/article/pii/ S0008622311000911

[9] Y. Liu, B. I. Yakobson, Cones, pringles, and grain boundary landscapes in graphene topology Nano Letters 10 (6) (2010) 2178-2183, pMID: 20481585. arXiv: http://dx.doi.org/10.1021/nl100988r doi : $10.1021 / \mathrm{nl} 100988 \mathrm{r}$ URL http://dx.doi.org/10.1021/nl100988r

[10] O. V. Yazyev, Y. P. Chen, Polycrystalline graphene and other twodimensional materials Nat. Nanotech. 9 (2014) 755-767. URL http://dx.doi.org/10.1038/nnano.2014.166

[11] A. W. Cummings, D. L. Duong, V. L. Nguyen, D. Van Tuan, J. Kotakoski, J. E. Barrios Vargas, Y. H. Lee, S. Roche, Charge transport in polycrystalline graphene: Challenges and opportunities Advanced Materials 26 (30) (2014) 5079-5094. doi : 10.1002/adma. 201401389 URL http://dx.doi.org/10.1002/adma.201401389

[12] T. Ma, Z. Liu, J. Wen, Y. Gao, X. Ren, H. Chen, C. Jin, X.-L. Ma, N. Xu, H.-M. Cheng, W. Ren, Tailoring the thermal and electrical transport properties of graphene films by grain size engineering Nature Communications 8 (2017) 14486. URL http://dx.doi.org/10.1038/ncomms 14486

[13] K. R. Hahn, C. Melis, L. Colombo, Thermal transport in nanocrystalline graphene investigated by approach-to-equilibrium molecular dynamics simulations. Carbon 96 (2016) $429-438$. doi:http://dx.doi.org/10.1016/j.carbon.2015.09.070 URL http://www.sciencedirect.com/science/article/pii/ S0008622315302888

[14] Z. Fan, L. F. C. Pereira, P. Hirvonen, M. M. Ervasti, K. R. Elder, D. Donadio, A. Harju, T. Ala-Nissila, Bimodal grain-size scaling of thermal transport in polycrystalline graphene from large-scale molecular dynamics simulations, Unpublished.

[15] A. Bagri, S.-P. Kim, R. S. Ruoff, V. B. Shenoy, Thermal transport across twin grain boundaries in polycrystalline graphene from nonequilibrium molecular dynamics simulations $\mid$ Nano Letters 11 (9) (2011) 3917-3921, pMID: 21863804. arXiv:http://dx.doi.org/ 10.1021/nl202118d doi:10.1021/nl202118d URL http://dx.doi.org/10.1021/nl202118d

[16] A. Cao, J. Qu, Kapitza conductance of symmetric tilt grain boundaries in graphene Journal of Applied Physics 111 (5) (2012) 053529. 
arXiv:http://dx.doi.org/10.1063/1.3692078 doi:10.1063/ 1.3692078

URL http://dx.doi.org/10.1063/1.3692078

[17] Y. Lu, J. Guo, Thermal transport in grain boundary of graphene by nonequilibrium greens function approach Applied Physics Letters 101 (4) (2012) 043112. arXiv:http://dx.doi.org/10.1063/1.4737653 doi:10.1063/1.4737653

URL http://dx.doi.org/10.1063/1.4737653

[18] A. Y. Serov, Z.-Y. Ong, E. Pop, Effect of grain boundaries on thermal transport in graphene Applied Physics Letters 102 (3) (2013) 033104. arXiv:http://dx.doi.org/10.1063/1.4776667 doi: 10.1063/1.4776667

URL http://dx.doi.org/10.1063/1.4776667

[19] P. Yasaei, A. Fathizadeh, R. Hantehzadeh, A. K. Majee, A. El-Ghandour, D. Estrada, C. Foster, Z. Aksamija, F. Khalili-Araghi, A. SalehiKhojin, Bimodal phonon scattering in graphene grain boundaries Nano Letters 15 (7) (2015) 4532-4540, pMID: 26035002. arXiv:http: //dx.doi.org/10.1021/acs.nanolett.5b01100 doi:10.1021/ acs.nanolett.5b01100

URL http://dx.doi.org/10.1021/acs.nanolett.5b01100

[20] P. Hirvonen, M. M. Ervasti, Z. Fan, M. Jalalvand, M. Seymour, S. M. Vaez Allaei, N. Provatas, A. Harju, K. R. Elder, T. Ala-Nissila, Multiscale modeling of polycrystalline graphene: A comparison of structure and defect energies of realistic samples from phase field crystal models Phys. Rev. B 94 (2016) 035414. doi:10.1103/PhysRevB.94.035414

URL https://link.aps.org/doi/10.1103/PhysRevB.94. 035414

[21] K. R. Elder, M. Katakowski, M. Haataja, M. Grant, Modeling elasticity in crystal growth Phys. Rev. Lett. 88 (2002) 245701. doi:10.1103/PhysRevLett.88.245701

URL https://link.aps.org/doi/10.1103/PhysRevLett.88. 245701

[22] K. R. Elder, M. Grant, Modeling elastic and plastic deformations in nonequilibrium processing using phase field crystals Phys. Rev. E 70 (2004) 051605. doi:10.1103/PhysRevE.70.051605

URL https://link.aps.org/doi/10.1103/PhysRevE.70. 051605

[23] K. Kim, Z. Lee, W. Regan, C. Kisielowski, M. F. Crommie, A. Zettl, Grain boundary mapping in polycrystalline graphene ACS Nano 5 (3) (2011) 2142-2146, pMID: 21280616. arXiv:http://dx.doi.org/ 10.1021/nn1033423 doi:10.1021/nn1033423

URL http://dx.doi.org/10.1021/nn1033423

[24] Z. Fan, T. Siro, A. Harju, Accelerated molecular dynamics force evaluation on graphics processing units for thermal conductivity calculations Computer Physics Communications 184 (5) (2013) 1414 - 1425. doi:http://dx.doi.org/10.1016/j.cpc.2013.01.008 URL http://www.sciencedirect.com/science/article/pii/ S0010465513000258

[25] Z. Fan, L. F. C. Pereira, H.-Q. Wang, J.-C. Zheng, D. Donadio, A. Harju, Force and heat current formulas for many-body potentials in molecular dynamics simulations with applications to \begin{tabular}{|l|lll}
\hline thermal conductivity calculations & Phys. Rev. B 92 (2015) 094301.
\end{tabular} doi:10.1103/PhysRevB.92.094301

URL https://link.aps.org/doi/10.1103/PhysRevB.92. 094301

[26] Z. Fan, W. Chen, V. Vierimaa, A. Harju, Efficient molecular dynamics simulations with many-body potentials on graphics processing units Computer Physics Communications 218 (2017) 10 - 16. doi:https://doi.org/10.1016/j.cpc.2017.05.003

URL http://wWw.sciencedirect.com/science/article/pii/ S0010465517301339

[27] J. Tersoff, Modeling solid-state chemistry: Interatomic potentials for \begin{tabular}{|l|l|l|}
\hline multicomponent systems & Phys. Rev. B 39 (1989) 5566-5568. doi :
\end{tabular} 10.1103/PhysRevB.39.5566

URL https://link.aps .org/doi/10.1103/PhysRevB.39.5566

[28] L. Lindsay, D. A. Broido, Optimized tersoff and brenner empirical potential parameters for lattice dynamics and phonon thermal transport in carbon nanotubes and graphene Phys. Rev. B 81 (2010) 205441. doi:10.1103/PhysRevB.81.205441

URL https://link.aps.org/doi/10.1103/PhysRevB.81. 205441
[29] S. Nosé, A unified formulation of the constant temperature molecular dynamics methods The Journal of Chemical Physics 81 (1) (1984) 511-519. arXiv:http://dx.doi.org/10.1063/1.447334 doi:10.1063/1. 447334 URL http://dx.doi.org/10.1063/1.447334

[30] W. G. Hoover, Canonical dynamics: Equilibrium phase-space distributions Phys. Rev. A 31 (1985) 1695-1697. doi:10.1103/PhysRevA. 31.1695 URL https://link.aps.org/doi/10.1103/PhysRevA.31.1695

[31] G. J. Martyna, M. L. Klein, M. Tuckerman, Nosé-hoover chains: The canonical ensemble via continuous dynamics The Journal of Chemical Physics 97 (4) (1992) 2635-2643. arXiv: http://dx.doi.org/10. 1063/1.463940 doi:10.1063/1.463940 URL http://dx.doi.org/10.1063/1.463940

[32] W. C. Swope, H. C. Andersen, P. H. Berens, K. R. Wilson, A computer simulation method for the calculation of equilibrium constants for the formation of physical clusters of molecules: Application to small water clusters The Journal of Chemical Physics 76 (1) (1982) 637-649. arXiv:http://dx.doi.org/10.1063/1.442716 doi:10.1063/1. 442716 URL http://dx.doi.org/10.1063/1.442716

[33] E. S. Landry, A. J. H. McGaughey, Thermal boundary resistance predictions from molecular dynamics simulations and theoretical calculations Phys. Rev. B 80 (2009) 165304. doi:10.1103/PhysRevB.80.165304 URL https://link.aps.org/doi/10.1103/PhysRevB.80. 165304

[34] A. Rajabpour, S. M. V. Allaei, F. Kowsary, Interface thermal resistance and thermal rectification in hybrid graphene-graphane nanoribbons: A nonequilibrium molecular dynamics study Applied Physics Letters 99 (5) (2011) 051917. arXiv:http://dx.doi.org/10.1063/1.3622480 doi: $10.1063 / 1.3622480$ URL http://dx.doi.org/10.1063/1.3622480

[35] K. Gordiz, S. M. V. Allaei, Thermal rectification in pristine-hydrogenated carbon nanotube junction: A molecular dynamics study Journal of Applied Physics 115 (16) (2014) 163512. arXiv:http://dx.doi.org/ 10.1063/1.4873124 doi:10.1063/1.4873124 URL http://dx.doi.org/10.1063/1.4873124

[36] J. K. Bohrer, K. Schrer, L. Brendel, D. E. Wolf, Thermal resistance of twist boundaries in silicon nanowires by nonequilibrium molecular dynamics AIP Advances 7 (4) (2017) 045105. arXiv:http://dx.doi. org/10.1063/1.4979982 doi:10.1063/1.4979982 URL http://dx.doi.org/10.1063/1.4979982

[37] K. Sääskilahti, J. Oksanen, J. Tulkki, S. Volz, Role of anharmonic phonon scattering in the spectrally decomposed thermal conductance at planar interfaces Phys. Rev. B 90 (2014) 134312. doi:10.1103/PhysRevB.90.134312

URL https://link.aps.org/doi/10.1103/PhysRevB.90. 134312

[38] K. Sääskilahti, J. Oksanen, S. Volz, J. Tulkki, Frequency-dependent phonon mean free path in carbon nanotubes from nonequilib-

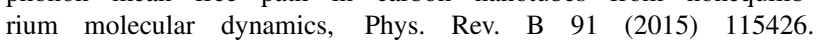
doi:10.1103/PhysRevB.91.115426

URL https://link.aps.org/doi/10.1103/PhysRevB.91. 115426

[39] K. Sääskilahti, J. Oksanen, J. Tulkki, A. J. H. McGaughey, S. Volz, Vibrational mean free paths and thermal conductivity of amorphous silicon from non-equilibrium molecular dynamics simulations AIP Advances 6 (12) (2016) 121904. arXiv: http://dx.doi.org/10.1063/ 1.4968617 doi:10.1063/1.4968617 URL http://dx.doi.org/10.1063/1.4968617

[40] Y. Zhou, X. Zhang, M. Hu, Quantitatively analyzing phonon spectral contribution of thermal conductivity based on nonequilibrium molecular dynamics simulations. I. From space Fourier transform Phys. Rev. B 92 (2015) 195204. doi:10.1103/PhysRevB.92.195204

URL https://link.aps.org/doi/10.1103/PhysRevB.92. 195204

[41] Y. Zhou, M. Hu, Quantitatively analyzing phonon spectral contribution of thermal conductivity based on nonequilibrium molecular dynamics simulations. II. From time Fourier transform Phys. Rev. B 92 (2015) 195205. doi:10.1103/PhysRevB.92.195205

URL https://link.aps.org/doi/10.1103/PhysRevB.92. 
195205

[42] Z. Fan, L. F. C. Pereira, P. Hirvonen, M. M. Ervasti, K. R. Elder, D. Donadio, T. Ala-Nissila, A. Harju, Thermal conductivity decomposition in two-dimensional materials: Application to graphene Phys. Rev. B 95 (2017) 144309. doi:10.1103/PhysRevB.95.144309

URL https://link.aps.org/doi/10.1103/PhysRevB.95. 144309

[43] T. Feng, W. Yao, Z. Wang, J. Shi, C. Li, B. Cao, X. Ruan, Spectral analysis of nonequilibrium molecular dynamics: Spectral phonon temperature and local nonequilibrium in thin films and across interfaces Phys. Rev. B 95 (2017) 195202. doi:10.1103/PhysRevB.95.195202

URL https://link.aps.org/doi/10.1103/PhysRevB.95. 195202

[44] J. M. Dickey, A. Paskin, Computer simulation of the lattice dynamics of solids. Phys. Rev. 188 (1969) 1407-1418. doi:10.1103/PhysRev. 188.1407

URL https://link.aps.org/doi/10.1103/PhysRev.188.1407

[45] E. Pop, V. Varshney, A. K. Roy, Thermal properties of graphene: Fundamentals and applications, MRS Bulletin 37 (12) (2012) 1273-1281. doi: $10.1557 / \mathrm{mrs} .2012 .203$

[46] J. E. Turney, A. J. H. McGaughey, C. H. Amon, Assessing the applicability of quantum corrections to classical thermal conductivity predictions Phys. Rev. B 79 (2009) 224305. doi: 10.1103/PhysRevB.79.224305 URL https://link.aps.org/doi/10.1103/PhysRevB.79. 224305

[47] T.-H. Liu, S.-C. Lee, C.-W. Pao, C.-C. Chang, Anomalous thermal transport along the grain boundaries of bicrystalline graphene nanoribbons from atomistic simulations Carbon 73 (2014) $432-442$. doi:http://dx.doi.org/10.1016/j.carbon.2014.03.005 URL http://www.sciencedirect.com/science/article/pii/ S0008622314002474

[48] C.-W. Nan, R. Birringer, D. R. Clarke, H. Gleiter, Effective thermal conductivity of particulate composites with interfacial thermal resistance Journal of Applied Physics 81 (10) (1997) 6692-6699. arXiv:http: //dx.doi.org/10.1063/1.365209 doi:10.1063/1.365209 URL http://dx.doi.org/10.1063/1.365209 